\title{
ON ALGEBRAIC VARIETIES OF $k$ DIMENSIONS IN SPACE OF $r$ DIMENSIONS
}

\author{
BY B. C. WONG
}

An algebraic $k$-dimensional variety $V_{k}$ which is the locus of $\infty^{k}$ points and not the locus of $\infty^{k-h} h$-spaces, where $h>0$, possesses numerous characteristics. Certain $2 k$ of these will be regarded as essential and all the others may be expressed in terms of them. Besides the order, $n$, of the variety, we shall define the other $2 k-1$ essential characteristics in the following manner.

Consider $V_{k}$ as belonging to an $r$-space $S_{r}$. A general $(r-k+t)$ space $S_{r-k+t}[1 \leqq t \leqq k]$ of $S_{r}$ intersects $V_{k}$ in a $V_{t}$. The $\infty^{2 t-1}$ tangent lines of this $V_{t}$ form a variety $W_{2 t}$ of $2 t$ dimensions. Let $j_{t}$ be the order of $W_{2 t}$, that is, the number of tangent lines of $V_{t}$ that meet a given $(r-k-t)$-space of $S_{r-k+t}$. If $V_{t}$ is projected upon a $(2 t-1)$-space of $S_{r-k+t}$, the projection will have $j_{t}$ pinch points.

Now an $(r-k+t-1)$-space of $S_{r-k+t}$ intersects the $k$-dimensional variety $V_{t}$ in a $V_{t-1}$. If $V_{t-1}$ possesses a conical point, we say that the $(r-k+t-1)$-space is tangent to $V_{t}$. The number of tangent $(r-k+t-1)$-spaces of $V_{t}$ passing through a given $(r-k+t-2)$-space of $S_{r-k+t}$ is finite. Denote this number by $m_{t}$. We say that $m_{t}$ is the class of $V_{t}$. Obviously, $m_{1} \equiv j_{1}$.

Thus, we have defined $2 k$ of the characteristics of the variety $V_{k}: n ; j_{1}, j_{2}, \cdots, j_{k} ; m_{1} \equiv j_{1}, m_{2}, \cdots, m_{k}$. We regard these as essential.

Our present knowledge of $k$-dimensional varieties is practically nil, except for the case where $k=2$ and for the case where the varieties are loci of $\infty^{1}(k-1)$-spaces. In this note our purpose is to call attention to the fact that, if $V_{k}$ is the complete intersection of $r-k$ hypersurfaces, $k-1$ of the $2 k$ essential characteristics can be expressed in terms of the remaining $k+1$. We find it convenient to express $m_{2}, m_{3}, \cdots, m_{k}$ in terms of $n$ and $j_{1}, j_{2}, \cdots, j_{k}$.

Let the $r-k$ hypersurfaces be of orders $n_{1}, n_{2}, \cdots, n_{r-k}$, respectively. Then $n=n_{1} n_{2} \cdots n_{r-k}$. By the methods of analytic 
geometry, we find that the values of the $j$ 's and $m$ 's are, writing $\alpha_{i}$ for $n_{i}-1$,

$$
\begin{aligned}
& i_{1}=n \sum \alpha_{1}, \quad j_{2}=n \sum \alpha_{1} \alpha_{2}, \cdots, \quad j_{k}=n \sum \alpha_{1} \alpha_{2} \cdots \alpha_{k} \text {; } \\
& m_{2}=n\left(\sum \alpha_{1}^{2}+\sum \alpha_{1} \alpha_{2}\right) \text {, } \\
& m_{3}=n\left(\sum \alpha_{1}^{3}+\sum \alpha_{1}^{2} \alpha_{2}+\sum \alpha_{1} \alpha_{2} \alpha_{3}\right) \text {, }
\end{aligned}
$$

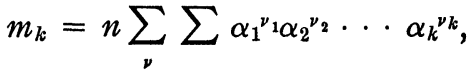

where

$$
\nu=\nu_{1}+\nu_{2}+\cdots+\nu_{k}=k .
$$

It is not difficult to see that if we eliminate the $\alpha$ 's from these equations, we have all the $m$ 's expressed in terms of $n$ and the $j$ 's. We shall give the values for a few of the $m$ 's:

$$
\begin{aligned}
n m_{2}= & j_{1}^{2}-n j_{2}, \\
n^{2} m_{3}= & j_{1}^{3}-2 n j_{1} j_{2}+n^{2} j_{3}, \\
n^{3} m_{4}= & j_{1}^{4}-3 n j_{1}^{2} j_{2}+n^{2} j_{2}^{2}+2 n^{2} j_{1} j_{3}-n^{3} j_{4}, \\
n^{4} m_{5}= & j_{1}{ }^{5}-4 n j_{1}^{3} j_{2}+3 n^{2} j_{1} j_{2}^{2}+3 n^{2} j_{1}^{2} j_{3}-2 n^{3} j_{2} j_{3} \\
& \quad-2 n^{3} j_{1} j_{4}+n^{4} j_{5}, \\
n^{5} m_{6}= & j_{1}^{6}-5 n j_{1}^{4} j_{2}+4 n^{2} j_{1}^{3} j_{3}+6 n^{2} j_{1}^{2} j_{2}^{2}-n^{3} j_{2}^{3}-6 n^{3} j_{1} j_{2} j_{3} \\
& \quad-3 n^{3} j_{1}^{2} j_{4}-2 n^{4} j_{3}^{2}+2 n^{4} j_{2} j_{4}+2 n^{4} j_{1} j_{5}-n^{5} j_{6} .
\end{aligned}
$$

The University of California 